\title{
A new strategy for vascular complications in young people with type 1 diabetes
}

M. Loredana Marcovecchio ${ }^{1}$, R. Neil Dalton ${ }^{2}$, Denis Daneman ${ }^{3}$, John Deanfield ${ }^{4}$, Timothy W. Jones $^{5}$, H. Andrew W. Neil ${ }^{6}$ and David B. Dunger ${ }^{1,7 *}$, on behalf of the Adolescent type 1 Diabetes cardio-renal Intervention Trial (AdDIT) study group

${ }^{1}$ Department of Paediatrics, University of Cambridge, Cambridge, UK

${ }^{2}$ Guy's and St Thomas' NHS Foundation Trust, London, UK

${ }^{3}$ Department of Paediatrics, The Hospital for Sick Children and University of Toronto, Toronto, Ontario, Canada

${ }^{4}$ Vascular Physiology Unit, Institute of Cardiovascular Science, University College, London, UK

${ }^{5}$ Telethon Kids Institute, University of Western Australia, Perth, Australia ${ }^{6}$ Oxford Centre for Diabetes, Endocrinology\& Metabolism, University of Oxford, Oxford, UK ${ }^{7}$ Wellcome Trust-MRC Institute of Metabolic Science, University of Cambridge, Cambridge, UK.

Word count: 3186

Figures: 5

*email:dbd25@cam.ac.uk 


\begin{abstract}
Diabetes vascular complications, including cardiovascular disease, diabetic nephropathy and retinopathy, have a negative effect on the long-term prognosis of young people with type 1 diabetes mellitus. Poor glycaemic control and consequent increased $\mathrm{HbA}_{1 \mathrm{c}}$ levels are major risk factors for the development of vascular complications. $\mathrm{HbA}_{1 \mathrm{c}}$ levels are the main focus of current management strategies, however, recommended target is rarely achieved in adolescents. Thus, a clear need exists for improved biomarkers to identify high risk young people at an early stage and to develop new intervention strategies. Evidence is accumulating that early increases in urinary albumin excretion could be predictive of adolescents with type 1 diabetes who are at an increased risk of developing vascular complications, independently of $\mathrm{HbA}_{1 \mathrm{c}}$ levels. These findings present an opportunity to move towards the personalized care of adolescents with type 1 diabetes, which takes into consideration changes in albumin excretion and other risk factors, in addition to $\mathrm{HbA}_{1 \mathrm{c}}$ levels.
\end{abstract}




\section{Introduction}

Type 1 diabetes mellitus (T1DM affects over 1 million young people (aged 0-20 years) worldwide and its incidence is increasing ${ }^{1-3}$. In Europe the incidence rate doubled between 1989 and 2013, with an overall annual increase of 3.4\% per year ${ }^{3}$. The principal causes of morbidity and mortality of T1DM are diabetic nephropathy and cardiovascular disease, which tend to occur 1-30 years after diagnosis ${ }^{4,5}$. In addition, sight threatening retinopathy has emerged as an important complication that occurs from the second decade after diagnosis 6 .

People with T1DM have decreased life expectancy compared to the background population ${ }^{7-10}$. Moreover, individuals who develop diabetes at a younger age have worse outcomes than those with an older age of onset ${ }^{11,12}$. In 2018, a US cohort study which used data derived from the National Health Interview Survey Linked Mortality files indicated that, although all-cause mortality substantially declined between 1988-1994 and 2010-2015 in adults with T1DM older than 44 years, mortality remained almost unchanged in younger people with T1DM (aged 20-44 years old) ${ }^{11}$. This observation has been further confirmed by the 2018 findings from the Swedish National Diabetes Register, which showed an excess mortality and cardiovascular disease risk in young adults (mean age: 29 years) with T1DM that was related to the age at onset ${ }^{12}$. The results emphasized that diagnosis before the age of 10 years was associated with a loss of life expectancy from the general population mean of 17.7 years in women and 14.2 years in men, as compared to 10.1 years in women and 9.4 years in men diagnosed between the ages of 26-30 years ${ }^{12}$.

The Swedish study also showed that age at diagnosis has a striking effect on cardiovascular outcomes. Individuals diagnosed with T1DM before the age of 10 years have a 30 -fold increased risk of coronary heart disease and acute myocardial infarction in early adulthood (mean age at follow up 29 years) compared to peers without diabetes ${ }^{12}$. These findings have generated considerable concerns for parents and those caring for young people with T1DM. Overall, the findings reiterate the need for improved methods to identify patients at risk of complications as well as for better screening for complications and treatment strategies. 
In this Perspectives, we discuss the potential effect of recent findings (between 2009 and 2018) from cohorts of adolescents with T1DM, on the role of urinary albumin excretion as a predictor of vascular complications. We then propose a new framework for patient risk stratification that is based on monitoring $A C R, \mathrm{HbA}_{1 \mathrm{c}}$ levels and other risk factors, to guide future recommendations for screening and interventions.

\section{Glycaemic control}

The risk of vascular complications.

Adolescence is a high risk period for the development of complications of T1DM, as optimal glycaemic control is difficult to achieve, due to the effect of hormonal changes and psychosocial factors ${ }^{13,14}$. The first signs of vascular complications appear during adolescence and subclinical manifestations are common, however, hard endpoints, such as overt proteinuria, proliferative retinopathy or cardiovascular events are rare ${ }^{15,16}$. Subclinical manifestations include early increases in urinary albumin excretion, changes in the retinal microvasculature and subclinical changes in large blood vessels, including increased arterial stiffness, aortic intima-media thickness (aIMT) and carotid intima-media thickness (cIMT) ${ }^{15}$ (Figure 1).

Glycaemic control is the main modifiable risk factor for vascular complications, which is clearly defined by the Diabetes Control and Complication Trial (DCCT) and the follow up study, the Epidemiology of Diabetes Interventions and Complications (EDIC) ${ }^{17,18}$. Only a small number (195 out of 1441) of adolescents (age 13-17 years) were included in the DCCT. In adolescents with T1DM, the beneficial effect of intensive therapy in reducing HbA1c levels was similar to the adult cohort. However, the overall levels of $\mathrm{HbA}_{1 \mathrm{c}}$ were around $1 \%$ higher in adolescents than those observed in adults ${ }^{17,19}$. The results of the DCCT and EDIC studies have continued to inform $\mathrm{HbA}_{1 \mathrm{c}}$ target levels proposed by the American Diabetes Association $(<7.5 \% \text { or } 58 \mathrm{mmol} \text { per } \mathrm{mol})^{20}$, the International Society for Pediatric and Adolescent Diabetes $(<7.0 \% \text { or } 53 \mathrm{mmol} \text { per } \mathrm{mol})^{21}$, the National Institute of Clinical Excellence $(<6.5 \% \text { or } 48 \mathrm{mmol} \text { per mol })^{22}$ and other organisations $\mathrm{s}^{23}$.

\section{Improvements in management.}

Ambitious targets for glycaemic control have been partly driven by considerable developments in the care and management of young people with T1DM ${ }^{24,25}$. The most 
important developments have been in the monitoring of day to day changes in glucose levels using continuous glucose monitoring systems, refinements in insulin delivery with new insulin analogues and the increasing use of insulin pump therapy to improve insulin delivery ${ }^{24}$. Since 2010 , further progress has been made with the introduction of closed loop insulin delivery (also known as artificial pancreas) that links glucose sensor data to a central algorithm, which improves the accuracy of insulin delivery ${ }^{26}$. Closed loop insulin delivery may increase time in the target range for glucose levels, thereby preventing hyperglycaemia and avoiding hypoglycaemia 27 .

Achieving the recommended glycaemic targets to reduce the risk of long-term complications remains a major challenge in adolescent populations with T1DM, as evidenced by data from the National Pediatric Diabetes Audit in the UK (28.9\% of adolescents achieve $\mathrm{HbA}_{1 \mathrm{c}}$ target levels of $<7.5 \%)^{28}$, the Australiasian Diabetes Data Network ( $27 \%$ of adolescents achieve $\mathrm{HbA}_{1 \mathrm{c}}$ target levels) ${ }^{29}$, and the T1D Exchange Clinical Registry in the USA (17-23\% of adolescents achieve $\mathrm{HbA}_{1 \mathrm{c}}$ target levels) ${ }^{30}$. These data raise the question as to whether, in addition to striving for improvements in $\mathrm{HbA}_{1 c}$ levels, we should do more to prevent complications in children and adolescents with T1DM.

\section{Age at diagnosis and duration}

The duration of diabetes is closely linked to risk of complications, however, several threads of evidence suggest that the age at diagnosis might also be an important non-modifiable risk factor $^{31-33}$. Cohort studies have highlighted a higher risk for early signs of vascular complications (for example, microalbuminuria and retinopathy) that are associated with an early age at diagnosis (that is, during childhood or adolescence), compared to a diagnosis during adulthood ${ }^{31,34}$. In the Oxford Regional Prospective Study (ORPS) cohort of children and adolescents with T1DM, the cumulative prevalence of microalbuminuria was $26 \%$ after 10 years duration and $51 \%$ after 19 years duration of diabetes ${ }^{31}$. By contrast, in a comparable adult cohort prevalence of microalbuminuria was $34 \%$ after 18 years duration of diabetes and exposure to similar levels of glycaemic control as in the ORPS cohort ${ }^{35}$. 
Interestingly, young people from the ORPS cohort who were diagnosed prior to puberty, between the ages of 0 to 10 years, showed a low prevalence of microalbuminuria until they reached puberty, thereafter microalbuminuria prevalence increased ${ }^{31}$. By contrast, individuals diagnosed during puberty showed a consistent increased cumulative prevalence of microalbuminuria ${ }^{31}$. Notably, the risk of developing retinopathy is reportedly higher in people diagnosed before the age of 14 years than in those diagnosed during adulthood ${ }^{34}$. These data suggest that a diagnosis of T1DM made during childhood and adolescence might be associated with different risk factors for the development of complications when compared to a later diagnosis made during adult life.

\section{Pubertal risk factors}

During the pubertal years ( 10-16years of age) several factors other than glycaemic control, related to growth and associated hormonal changes, can contribute to the development of the early signs of vascular complications $s^{33,36-38}$. These factors include abnormalities in the GH-insulin-like growth factors 1 axis, androgens levels in girls and other risk factors, such as high blood pressure, dyslipidaemia, obesity, insulin resistance, inflammatory markers, renal hyperfiltration, and a familial or genetic predisposition ${ }^{33,36}$.

In adolescents with T1DM, insulin resistance is a prominent feature, even in the absence of excess weight gain ${ }^{39}$. Moreover, insulin resistance can contribute to the increased risk of complications, such as microalbuminuria and abnormal cardiovascular markers ${ }^{38-42}$. Notably, a sexual dimorphism exists for the risk of complications during adolescence, with a higher percentage of girls developing microalbuminuria or retinopathy ${ }^{31,43}$. By contrast men have a higher risk of complications during adulthood ${ }^{35}$. Adolescent girls with microalbuminuria had higher testosterone levels when compared to matched control individuals without this complication ${ }^{44}$, suggesting that an increased androgen production by the ovary could contribute not only to the risk of polycystic ovary syndrome in girls with T1DM ${ }^{45}$, but also to the female predominance of the early signs of vascular complications. Environmental influences and dietary factors, such as low adherence to healthy diets, might also contribute to the risk of developing complications during puberty and represent potential targets for interventions ${ }^{36,46}$. 


\section{Albumin excretion as a biomarker}

Microalbuminuria is the most frequently studied marker of the vascular complications of $\mathrm{T} \mathrm{DM}^{47-49}$. Although originally described as an early marker of incident diabetic nephropathy, the presence of microalbuminuria has also been associated with an increased risk of cardiovascular disease and is identified as a significant determinant of mortality in adults with T1DM ${ }^{50}$.

\section{Adults with T1DM.}

Longitudinal studies have shown that, in the absence of microalbuminuria, mortality in adults with T1DM is similar to that in the general population, whereas it substantially increases in individuals with increased urinary albumin excretion that falls above the cutoff for microalbuminuria ${ }^{51,52}$. Meta-analyses from 2012 and 2015 indicate that even early increases in albumin excretion that fall within the normal range might predict renal and cardiovascular risk in adults with T1DM and also in adults from the general population ${ }^{53,54}$. Higher values of urinary albumin excretion within the normal have been directly associated with increased risk of stroke, hypertension, and early development of atherosclerosis, as compared to lower values within the normal range ${ }^{55}$. A potential factor linking increased albumin excretion with renal and cardiovascular disease is endothelial dysfunction, which in the context of diabetes might be the initiating pathology leading to tissue and organ damage ${ }^{56}$. Taken together these data suggest that in adults with T1DM urinary albumin excretion should be considered a continuous risk factor for both renal and cardiovascular complications.

Young people with T1DM.

Studies of adolescents with T1DM from longitudinal cohorts, such as the ORPS and Nephropathy Family Study (NFS), extended the findings from adult cohorts by providing longitudinal data from diagnosis that associate early increases in urinary albumin-creatinine ratios (ACR) with a subsequent increased risk for the development of microalbuminuria ${ }^{57,58}$ (Figure 2). In young people who developed microalbuminuria and subsequently progressed to diabetic nephropathy, gradual increases in ACR occurred from diagnosis, which 
accelerated during puberty ${ }^{57}$, and were associated with changes in blood pressure, lipid levels, and inflammatory markers ${ }^{59-61}$. In individuals with high $\mathrm{HbA}_{1 \mathrm{c}}$ levels and therefore poor glycaemic control, ACR subsequently progressed after puberty, whereas those with good glycaemic control showed relative reductions in ACR ${ }^{31}$. Importantly, in the ORPS and NFS cohorts, using an algorithm that allowed adjustments of ACR for age, sex and duration of diabetes, an ACR in the upper tertile of the normal range at the age of 10 to 16 years was highly predictive of patients who later developed nephropathy. This predictive ability held true in young people with both persistent and intermittent changes in $A C R^{58}$.

\section{The AdDIT studies}

Observations from early cohort studies on the relationship between adolescent urinary ACR and the risk of progression to diabetic nephropathy led to the design of the Adolescent Type 1 Diabetes cardio-renal Intervention Trial (AdDIT) studies ${ }^{62}$. The rationale for the trial was that adolescents (aged 10-16 years-old) who were in the highest tertile of ACR, but still fell below the cut-off for microalbuminuria, would probably be at greatest risk of complications and might benefit from 2-4 years treatment with angiotensin-converting enzyme (ACE) inhibitors and/or statins to protect against renal, retinal and cardiovascular complications ${ }^{63}$. These drugs are indicated for use in adults with T1DM who have microalbuminuria ${ }^{64}$. The AdDIT protocol also included an observational untreated cohort, who were deemed to be at low risk of complications based on an ACR falling in the low and middle tertiles; these adolescents were also followed over 2-4 years ${ }^{65}$ (Figure 3 ).

\section{ACR tertiles}

Screening for ACR in 4,407 adolescents with T1DM for the AdDIT studies confirmed the utility of a risk stratification system that is based on ACR tertiles. The tertiles were calculated from two sets of three consecutive early morning urine samples collected at least 1 month apart. The median (interquatile ranges) unadjusted ACR levels were 0.52 (0.430.62) $\mathrm{mg}$ per mmol for the lower tertile, $0.71(0.59-0.85) \mathrm{mg}$ per $\mathrm{mmol}$ for the middle and 1.24 (0.91-1.82) mg per mmol for the upper tertile ${ }^{65}$. For each study participant, ACR results were averaged on the log ACR scale and then standardised for sex, age and the duration of diabetes, using a linear regression equation derived the ORPS-NFS studies ${ }^{58}$. This led to a 
cut-point $>\log 1.2$ to define the upper ACR tertile, between $0.8-1.2$ to define the middle tertile and $<0.8$ to define the lower tertile.

In the AdDIT study, at baseline, adolescents in the highest tertile for ACR showed renal hyperfiltration, dyslipidaemia, early retinal vessel abnormalities and increased pulse wave velocity ${ }^{65,66}$. In a subgroup of the AdDIT cohort, where data on aIMT and autonomic function measures were available, a higher ACR at baseline was also associated with thicker alMT and the presence of markers of autonomic dysfunction ${ }^{67,68}$. However, despite these substantial differences in early vascular markers there were minimal differences in $\mathrm{HbA}_{1 \mathrm{c}}$ levels across the ACR tertiles, which suggests that renal and cardiovascular risk factors may be partly independent of glycaemic control ${ }^{65}$.

The results of both the AdDIT Intervention and AdDIT Observational studies have now been published $^{63,65,66,69}$ and it is important to consider how these results could influence screening and intervention strategies in high-risk and low-risk adolescents with T1DM.

\section{AdDIT Intervention study}

As previously mentioned, in adults with T1DM, the presence of microalbuminuria is a general indication for intervention with ACE inhibitors or angiotensin receptor blockers and also for consideration of statin therapy ${ }^{64}$. On the basis of the published data in adults with T1DM ${ }^{70}$, we predicted that intervention with ACE inhibitors might reduce the incidence of microalbuminuria in adolescents with T1DM. In addition, on the basis of positive results in young people with familial hypercholesterolaemia ${ }^{71}$, we also expected that statins would reduce exposure to hyperlipidaemia in adolescents with $\mathrm{T}_{1 D M^{62}}$. In the AdDIT intervention study, both these predictions proved to be true and both drugs were well tolerated during adolescence with very few adverse effects ${ }^{63}$.

In the adolescent AdDIT intervention cohort with ACR in the upper tertile, statin treatment resulted in substantial decreases in total cholesterol, LDL cholesterol and non-HDL cholesterol, the Apolipoprotein-B-Apolipoprotein-A ratio and high-sensitivity C-reactive protein (hsCRP) ${ }^{63}$, which might lead to a long-term reduction of cardiovascular risk. In 
addition, adolescents with T1DM from the AdDIT cohort who were treated with ACE inhibitors showed a trend towards a reduction in the prevalence of microalbuminuria, including cases of intermittent microalbuminuria ${ }^{63}$. This intervention could therefore lead to a long-term reduced risk of progression to diabetic nephropathy and prevention of cardiovascular disease $^{72}$. Participant adherence to ACE inhibitors and statins during the AdDIT trial was 75$80 \%{ }^{63}$, which is well above the average adherence in other trials with adolescent populations ${ }^{73}$, and both drugs were safe. These results are encouraging and suggest that statins and ACE inhibitors might be used more widely in individuals with T1DM from a younger age.

Importantly, the AdDIT intervention study failed to show an effect on the primary outcome, which was whether either ACE inhibitors or statins alone or in combination could reduce the baseline ACR in adolescents with T1DM and thus potentially reduce the ACR-associated risk for progression to diabetic nephropathy and cardiovascular disease ${ }^{63}$. In retrospect, this primary outcome was potentially naïve, as subsequent analysis of the AdDIT data suggests that the inherent risk of complications that is associated with high baseline ACR may be more closely related to genetic or environmental factors, which are unlikely to be influenced by these drugs ${ }^{69}$. The lack of effect of ACE inhibitors and statins in the AdDIT population on the primary outcome alongside with the known modulatory effect of other factors on albumin excretion during puberty $\mathrm{y}^{33,36,37}$, suggest that additional interventions might be necessary to impact on other mechanisms that are implicated in the pathogenesis of vascular complications during adolescence. These interventions could include new drugs as well as lifestyle changes to control weight gain and improve insulin sensitivity.

\section{AdDIT observational cohort.}

Subsequent study of the observational AdDIT cohort and those participants in the interventional cohort who were randomised to the placebo arm provided further insights into the predictive value of $A C R^{69}$. During the 2 to 4 years observational period, we noted substantial differences between adolescence participants with a high baseline ACR compared to those with a low baseline ACR (falling in the middle and lower tertiles Individuals with high baseline ACR were more likely to develop microalbuminuria but also 
more marked increases in cIMT, hsCRP and systolic blood pressure, which are indicative of future risk for both diabetic nephropathy and cardiovascular disease ${ }^{69}$. Notably, the greatest risk for progression of retinopathy was also observed in those in the highest tertile of $A C R^{74}$. Importantly, all these observations were partly independent of $\mathrm{HbA}_{1 c} 69,74$, which suggests that other pathogenic mechanisms in addition to effect of glycaemic control are responsible for diabetes vascular complications (Figure 4).

Together, these data suggest that repeated measures of ACR between the ages of 10-16 years could contribute additional prognostic information for young people with early onset T1DM. In the future, these findings could lead to precision medicine approaches to intensifying therapy based on $\mathrm{HbA}_{1 \mathrm{c}}$ levels and additional potential markers of the risk of complications such as ACR.

\section{A change in risk assessment?}

\section{Current guidelines.}

During adolescence the primary focuses of management the of risk of diabetes complications are improvements in glycaemic control and decreases in $\mathrm{HbA}_{1 c}{ }^{20,21}$, although the recommended target levels for $\mathrm{Hb}_{1} \mathrm{~A}_{1}$ are difficult to achieve ${ }^{29,30}$. Current guidelines also recommend regular screening for microalbuminuria (as a marker of incident nephropathy) and dyslipidaemia and hypertension (as markers of potential cardiovascular disease) from the ages of 10 to 11 years ${ }^{20,21}$. However, the current recommendations for appropriate interventions are still vague as there are few published outcome data.

The various existing clinical guidelines that recommend screening during adolescence for microalbuminuria differ only slightly in the advised age at screening, however, current guidelines only broadly describe the method of screening and interpretation of the results ${ }^{20-}$ 22. In the various guidelines, microalbuminuria is usually defined as two out of three urine collections that exceed a sex-specific value of ACR. Only individuals who are classified as having developed microalbuminuria are subsequently investigated for evidence of abnormalities in ambulatory blood pressure and may be started an ACE inhibitor ${ }^{20,21}$. 


\section{A new proposed approach.}

Data from ORPS and AdDIT suggest that the above described approach suggested in clinical guidelines for detecting or excluding incident nephropathy may not identify all young people at risk for diabetic nephropathy, cardiovascular disease and retinopathy. Urinary albumin excretion is highly variable and the single measurement made in three urines every year might fail to detect people who have increasing albumin excretion. These subjects with increasing albumin excretion need to be identified through repeated measures of ACR. The AdDIT results suggest that accurately tracking urinary albumin excretion using two sets of three repeated measurements of ACR annually from as early as 10 years of age, can help in identifying those adolescents with increased risk for future complications ${ }^{69}$. These data could be used alongside $\mathrm{HbA}_{1 \mathrm{c}}$ levels to determine the relative risk for complications, which could then be used to influence management. Those adolescents with a rapidly increasing ACR (defined as three consecutive ACR and poor glycaemic control would be the priority targets for intensive insulin therapy but also potentially for treatment with ACE inhibitors or angiotensin receptor blockers. Data from the AdDIT trial have also provided support for the wider use of statins in young people (>10 years of age) with T1DM in whom high lipid levels are detected. The studies provided reassuring safety data for this patient population, which are similar to those previously reported in children and adolescents with familial hypercholesterolemia ${ }^{75}$. Furthermore, these high risk young individuals might be potential candidates for new reno-protective drugs, such as Sodium-glucose Cotransporter2 inhibitors, which have shown promising results in terms of cardio-renal outcomes in adults with diabetes ${ }^{76}$.

We plan further analyses of the large AdDIT dataset to refine the ACR-based algorithm for the identification of adolescents who are at higher risk of renal, retinal and cardiovascular outcomes. We will explore interactions between $\mathrm{HbA}_{1 \mathrm{c}}$ and other biomarkers, such as lipids, blood pressure, BMI, glomerular filtration rate, inflammatory markers and new biomarkers emerging from ongoing 'omics' studies. Further follow up of the AdDIT cohort during adult life will also provide valuable information on the rates of long-term complications and hard endpoints and how these outcomes might by influenced by early interventions during the critical pubertal years. Even small reductions in $\mathrm{HbA}_{1 c}$, blood pressure, lipid levels, 
inflammatory markers and the rate of microalbuminuria during the AdDIT trial period could lead to longer-term cardio-renal protection.

\section{Conclusions}

Data from historical cohorts of children and adolescents with T1DM and the more recent AdDIT cohort provide evidence that variation in urinary albumin excretion, in patients aged 10-16 years, can predict future risk for vascular complications. These findings support the concept that risk stratification using ACR during early adolescence might be critical for the early identification of patients who are at risk of developing renal, retinal and cardiovascular complications.

In our opinion, detailed assessment and tracking of ACR, considered together with other risk factors during adolescence could be used in addition to $\mathrm{HbA}_{1 \mathrm{c}}$ to identify young people who are most at risk. This information might be used to guide future management and support prevention strategies in adolescents with T1DM based on precision medicine. 


\section{Acknowledgements}

The authors acknowledge the support of Diabetes UK, the Juvenile Diabetes Research Foundation, the British Heart Foundation, the JDRF- Canadian Clinical Trial Network (CCTN), the Canadian Diabetes Association and the Heart and Stroke Foundation Canada, which all contributed funding for the AdDIT studies

\section{AdDIT study group}

In the UK: Acerini C. (University of Cambridge, Cambridge, UK), Ackland F. (Northampton General Hospital, Northampton, UK), Anand B. (West Suffolk Hospital, NHS Foundation Trust, Bury St Edmunds, UK), Barrett T. (Birmingham Children's Hospital and University of Birmingham, Birmingham, UK), Birrell V. (James Cook Hospital, South Tees Hospitals NHS Foundation Trust, Middlesbrough, UK), Campbell F. (Leeds General Infirmary, The Leeds Teaching Hospitals NHS Trust, Leeds, UK), Charakida M. (King's College London, London, UK), Cheetham T. (Royal Victoria Infirmary, The Newcastle upon Tyne Hospitals NHS Foundation Trust and Newcastle University, Newcastle, UK), Chiesa T. (University College London, London, UK), John Deanfield (University College London, London, UK), Cooper C. (Stepping Hill Hospital, Stockport NHS Foundation Trust, Stockport, UK), Doughty I. (Royal Manchester Children's Hospital, Manchester, UK), Dutta A. (Stoke Mandeville Hospital, Aylesbury, UK), Edge J. (John Radcliffe Hospital, Oxford, UK), Gray A. (University of Oxford, Oxford, UK), Hamilton-Shield J. (University of Bristol and University Hospitals Bristol NHS Foundation Trust, Bristol, UK), Mann N. (Royal Berkshire Hospital, Reading, UK), Marcovecchio M.L. (University of Cambridge, Cambridge, UK), Marshall S. (University Newcastle), Rayman G. (Ipswich Hospitals NHS Trust, Ipswich, UK), Robinson J.M. (Royal Albert Edward Infirmary, Wrightington, Wigan and Leigh NHS Foundation Trust, Wigan, UK), Russell-Taylor M (Wycombe Hospital, Buckingham Healthcare NHS Trust, High Wycombe, UK), Sankar V. (Royal Bolton Hospital, Bolton NHS Foundation Trust, Bolton, UK), Smith A. (Northampton General Hospital, Northampton, UK), Thalange N. (Norfolk and Norwich University Hospitals NHS Foundation Trust, Norwich, UK), Yaliwal C. (Royal Berkshire Hospital, Reading, UK).

In Australia: Benitez-Aguirre P. (The Children's Hospital at Westmead and University of Sydney, Sydney, NSW, Australia), Cameron F. (Royal Children's Hospital, Murdoch Children's Research Institute and The University of Melbourne, Melbourne, VIC, Australia), Cotterill A. (University of Queensland, Brisbane, QLD, Australia), Couper J. (Women's and Children's Hospital and University of Adelaide, Adelaide, SA, Australia), Craig M. (The Children's Hospital at Westmead, University of Sydney, and University of New South Wales, Sydney, NSW, Australia), Davis E. (Princess Margaret Hospital for Children and University of Western Australia, Perth, WA, Australia), Donaghue K. (The Children's Hospital at Westmead and University of Sydney, Sydney, NSW, Australia), Jones T.W. (Princess Margaret Hospital for Children and University of Western Australia, Perth, WA, Australia), Bruce King (University of Newcastle, Newcastle, NSW, Australia), Verge C. (Sydney Children's Hospital and University of New South Wales, Sydney, NSW, Australia), Bergman P. (Monash Children's Hospital, Clayton, VIC, Australia), Rodda C. (University of Melbourne, Melbourne, VIC, Australia). 
In Canada: Clarson C. (London Health Sciences Centre and Western University, London, ON, Canada); Curtis J. (The Hospital for Sick Children and University of Toronto, Toronto, ON, Canada), Daneman D., (The Hospital for Sick Children and University of Toronto, Toronto. ON, Canada), Mahmud F. (The Hospital for Sick Children and University of Toronto, Toronto, ON, Canada); Sochett E. (The Hospital for Sick Children and University of Toronto, Toronto, ON, Canada).

\section{Authors contribution}

All authors researched data for the article. D.B.D., D.D., J.D., R.N.D and T.J. provided a substantial contribution to discussion of content. M.L.M. and D.B.D. wrote the article. All authors reviewed and edited the manuscript before submission.

\section{Publisher's note}

Springer Nature remains neutral with regard to jurisdictional claims in published maps and institutional affiliations.

Competing interestThe authors declare no competing interests. 


\section{References}

1. IDF Diabetes Atlas. (2017). Available at: http://www.diabetesatlas.org/.

2. Mayer-Davis, E. J. et al. Incidence Trends of Type 1 and Type 2 Diabetes among Youths, 2002-2012. N. Engl. J. Med. 376, 1419-1429 (2017).

3. Patterson, C. C. et al. Trends and cyclical variation in the incidence of childhood type 1 diabetes in 26 European centres in the 25 year period 1989-2013: a multicentre prospective registration study. Diabetologia 62, 408-417 (2019).

4. Secrest, A. M., Becker, D. J., Kelsey, S. F., Laporte, R. E. \& Orchard, T. J. Cause-specific mortality trends in a large population-based cohort with long-standing childhoodonset type 1 diabetes. Diabetes 59, 3216-22 (2010).

5. Bjornstad, P., Donaghue, K. C. \& Maahs, D. M. Macrovascular disease and risk factors in youth with type 1 diabetes: time to be more attentive to treatment? Lancet Diabetes Endocrinol. 6, 809-820 (2018).

6. Barrett, E. J. et al. Diabetic Microvascular Disease: An Endocrine Society Scientific Statement. J. Clin. Endocrinol. Metab. 102, 4343-4410 (2017).

7. Patterson, C. C. et al. Early mortality in EURODIAB population-based cohorts of type 1 diabetes diagnosed in childhood since 1989. Diabetologia 50, 2439-42 (2007).

8. Livingstone, S. J. et al. Estimated life expectancy in a Scottish cohort with type 1 diabetes, 2008-2010. JAMA 313, 37-44 (2015).

9. Petrie, D. et al. Recent trends in life expectancy for people with type 1 diabetes in Sweden. Diabetologia 59, 1167-76 (2016).

10. Huo, L., Harding, J. L., Peeters, A., Shaw, J. E. \& Magliano, D. J. Life expectancy of type 1 diabetic patients during 1997-2010: a national Australian registry-based cohort study. Diabetologia 59, 1177-85 (2016).

11. Gregg, E. W. et al. Trends in cause-specific mortality among adults with and without diagnosed diabetes in the USA: an epidemiological analysis of linked national survey and vital statistics data. Lancet 391, 2430-2440 (2018).

12. Rawshani, A. et al. Excess mortality and cardiovascular disease in young adults with type 1 diabetes in relation to age at onset: a nationwide, register-based cohort study. Lancet 392, 477-486 (2018).

13. Cameron, F. J. \& Wherrett, D. K. Care of diabetes in children and adolescents: controversies, changes, and consensus. Lancet 385, 2096-2106 (2015).

14. Cameron, F. J., Garvey, K., Hood, K. K., Acerini, C. L. \& Codner, E. ISPAD Clinical Practice Consensus Guidelines 2018: Diabetes in adolescence. Pediatr. Diabetes 19, 250-261 (2018).

15. Marcovecchio, M. L., Tossavainen, P. H. \& Dunger, D. B. Status and rationale of renoprotection studies in adolescents with type 1 diabetes. Pediatr. Diabetes 10, 347-355 (2009).

16. Sauder, K. A. et al. Co-occurrence of early diabetes-related complications in adolescents and young adults with type 1 diabetes: an observational cohort study. Lancet Child Adolesc. Heal. 3, 35-43 (2019).

17. Diabetes Control and Complications Trial Research group. The Effect of Intensive Treatment of Diabetes on the Development and Progression of Long-Term Complications in Insulin-Dependent Diabetes Mellitus. N. Engl. J. Med. 329, 977-986 (1993).

18. Subramanian, S. \& Hirsch, I. B. Intensive Diabetes Treatment and Cardiovascular Outcomes in Type 1 Diabetes Mellitus. Endocrinol. Metab. Clin. North Am. 47, 65-79 
(2018).

19. Diabetes Control and Complications Trial Research group. Effect of intensive diabetes treatment on the development and progression of long-term complications in adolescents with insulin-dependent diabetes mellitus: Diabetes Control and Complications Trial. J. Pediatr. 125, 177-188 (1994).

20. American Diabetes Association. 13. Children and Adolescents: Standards of Medical Care in Diabetes-2019. Diabetes Care 42, S148-S164 (2019).

21. Donaghue, K. C. et al. ISPAD Clinical Practice Consensus Guidelines 2018: Microvascular and macrovascular complications in children and adolescents. Pediatr. Diabetes 19, 262-274 (2018).

22. Diabetes (type 1 and type 2 ) in children Diabetes (type 1 and type 2 ) in children and young people: diagnosis and oung people: diagnosis and management management NICE guideline. (2015). Available at https://www.nice.org.uk/guidance/ng18

23. Wherrett, D. K. et al. Type 1 Diabetes in Children and Adolescents. Can. J. Diabetes 42, S234-S246 (2018).

24. Tauschmann, M. \& Hovorka, R. Technology in the management of type 1 diabetes mellitus - current status and future prospects. Nat. Rev. Endocrinol. 14, 464-475 (2018).

25. Prahalad, P., Tanenbaum, M., Hood, K. \& Maahs, D. M. Diabetes technology: improving care, improving patient-reported outcomes and preventing complications in young people with Type 1 diabetes. Diabet. Med. 35, 419-429 (2018).

26. Hovorka, R. et al. Manual closed-loop insulin delivery in children and adolescents with type 1 diabetes: a phase 2 randomised crossover trial. Lancet (London, England) 375, 743-51 (2010).

27. Bekiari, E. et al. Artificial pancreas treatment for outpatients with type 1 diabetes: systematic review and meta-analysis. BMJ 361, k1310 (2018).

28. National Paediatric Diabetes Audit 2016-2017 -Care Processes and Outcomes. Available at: https://www.rcpch.ac.uk/sites/default/files/201807/npda_annual_report_2016_-_2017_april_2018_final_updated_3.pdf.

29. Phelan, H. et al. The Australasian Diabetes Data Network: first national audit of children and adolescents with type 1 diabetes. Med. J. Aust. 206, 121-125 (2017).

30. Miller, K. M. et al. Current state of type 1 diabetes treatment in the U.S.: updated data from the T1D Exchange clinic registry. Diabetes Care 38, 971-8 (2015).

31. Amin, R. et al. Risk of microalbuminuria and progression to macroalbuminuria in a cohort with childhood onset type 1 diabetes: prospective observational study. BMJ 336, 697-701 (2008).

32. Cho, Y. H. et al. Microvascular complications assessment in adolescents with 2- to 5-yr duration of type 1 diabetes from 1990 to 2006. Pediatr. Diabetes 12, 682-689 (2011).

33. Dunger, D. B. Banting Memorial Lecture 2016 Reducing lifetime risk of complications in adolescents with Type 1 diabetes. Diabet. Med. 34, 460-466 (2017).

34. Hietala, K. et al. Age at onset and the risk of proliferative retinopathy in type 1 diabetes. Diabetes Care 33, 1315-9 (2010).

35. Hovind, P. et al. Predictors for the development of microalbuminuria and macroalbuminuria in patients with type 1 diabetes: inception cohort study. $B M J \mathbf{3 2 8}$, 1105 (2004).

36. Cho, Y. H., Craig, M. E. \& Donaghue, K. C. Puberty as an accelerator for diabetes complications. Pediatr. Diabetes 15, 18-26 (2014). 
37. Lane, P. H. Diabetic kidney disease: impact of puberty. Am. J. Physiol. Physiol. 283, F589-F600 (2002).

38. Amin, R. et al. Increasing urine albumin excretion is associated with growth hormone hypersecretion and reduced clearance of insulin in adolescents and young adults with type 1 diabetes: the Oxford Regional Prospective Study. Clin. Endocrinol. (Oxf). 62, 137-44 (2005).

39. Nadeau, K. J. et al. Insulin Resistance in Adolescents with Type 1 Diabetes and Its Relationship to Cardiovascular Function. J. Clin. Endocrinol. Metab. 95, 513-521 (2010).

40. Cree-Green, M. et al. Delayed Skeletal Muscle Mitochondrial ADP Recovery in Youth With Type 1 Diabetes Relates to Muscle Insulin Resistance. Diabetes 64, 383-392 (2015).

41. Bjornstad, P. et al. Insulin sensitivity is an important determinant of renal health in adolescents with type 2 diabetes. Diabetes Care 37, 3033-9 (2014).

42. Bjornstad, P. et al. Metformin Improves Insulin Sensitivity and Vascular Health in Youth With Type 1 Diabetes Mellitus. Circulation 138, 2895-2907 (2018).

43. Benitez-Aguirre, P. et al. Sex differences in retinal microvasculature through puberty in type 1 diabetes: are girls at greater risk of diabetic microvascular complications? Invest. Ophthalmol. Vis. Sci. 56, 571-7 (2015).

44. Amin, R. et al. Low IGF-I and elevated testosterone during puberty in subjects with type 1 diabetes developing microalbuminuria in comparison to normoalbuminuric control subjects: the Oxford Regional Prospective Study. Diabetes Care 26, 1456-61 (2003).

45. Codner, E. \& Cassorla, F. Puberty and Ovarian Function in Girls with Type 1 Diabetes Mellitus. Horm. Res. Paediatr. 71, 12-21 (2009).

46. Costacou, T. et al. Dietary Patterns Over Time and Microalbuminuria in Youth and Young Adults With Type 1 Diabetes: The SEARCH Nutrition Ancillary Study. Diabetes Care 41, 1615-1622 (2018).

47. Parving, H.-H., Persson, F. \& Rossing, P. Microalbuminuria: A parameter that has changed diabetes care. Diabetes Res. Clin. Pract. 107, 1-8 (2015).

48. Viberti, G. C. et al. Microalbuminuria as a predictor of clinical nephropathy in insulindependent diabetes mellitus. Lancet (London, England) 1, 1430-2 (1982).

49. Deckert, T., Feldt-Rasmussen, B., Borch-Johnsen, K., Jensen, T. \& Kofoed-Enevoldsen, A. Albuminuria reflects widespread vascular damage. The Steno hypothesis. Diabetologia 32, 219-26 (1989).

50. Rossing, P., Hougaard, P., Borch-Johnsen, K. \& Parving, H. H. Predictors of mortality in insulin dependent diabetes: 10 year observational follow up study. BMJ 313, 779-84 (1996).

51. Orchard, T. J., Secrest, A. M., Miller, R. G. \& Costacou, T. In the absence of renal disease, 20 year mortality risk in type 1 diabetes is comparable to that of the general population: a report from the Pittsburgh Epidemiology of Diabetes Complications Study. Diabetologia 53, 2312-2319 (2010).

52. Groop, P.-H. et al. The presence and severity of chronic kidney disease predicts allcause mortality in type 1 diabetes. Diabetes 58, 1651-8 (2009).

53. Fox, C. S. et al. Associations of kidney disease measures with mortality and end-stage renal disease in individuals with and without diabetes: a meta-analysis. Lancet $\mathbf{3 8 0 ,}$ 1662-1673 (2012). 
54. Matsushita, K. et al. Estimated glomerular filtration rate and albuminuria for prediction of cardiovascular outcomes: a collaborative meta-analysis of individual participant data. lancet. Diabetes Endocrinol. 3, 514-25 (2015).

55. Abdelhafiz, A. H., Ahmed, S. \& El Nahas, M. Microalbuminuria: Marker or Maker of Cardiovascular Disease. Nephron Exp. Nephrol. 119, e6-e10 (2011).

56. Schalkwijk, C. G. \& Stehouwer, C. D. A. Vascular complications in diabetes mellitus: the role of endothelial dysfunction. Clin. Sci. (Lond). 109, 143-59 (2005).

57. Schultz, C. J., Neil, H. A., Dalton, R. N., Dunger, D. B. \& Oxforn Regional Prospective Study Group. Risk of nephropathy can be detected before the onset of microalbuminuria during the early years after diagnosis of type 1 diabetes. Diabetes Care 23, 1811-5 (2000).

58. Dunger, D. B. et al. Can we identify adolescents at high risk for nephropathy before the development of microalbuminuria? Diabet. Med. 24, 131-6 (2007).

59. Marcovecchio, M. L. et al. Reduced endogenous secretory receptor for advanced glycation end products (esRAGE) in young people with Type 1 diabetes developing microalbuminuria. Diabet. Med. 26, 815-819 (2009).

60. Marcovecchio, M. L. et al. Prevalence of abnormal lipid profiles and the relationship with the development of microalbuminuria in adolescents with type 1 diabetes.

Diabetes Care 32, 658-663 (2009).

61. Marcovecchio, M. L. et al. Ambulatory blood pressure measurements are related to albumin excretion and are predictive for risk of microalbuminuria in young people with type 1 diabetes. Diabetologia 52, (2009).

62. Adolescent type 1 Diabetes Cardio-renal Intervention Trial (AdDIT). BMC Pediatr. 9, 79 (2009).

63. Marcovecchio, M. L. et al. ACE Inhibitors and Statins in Adolescents with Type 1 Diabetes. N. Engl. J. Med. 377, 1733-1745 (2017).

64. NICE. Type 1 diabetes in adults: diagnosis and management. 2016 Available at: https://www.nice.org.uk/guidance/ng17/chapter/1-recommendations\#managingcomplications.

65. Marcovecchio, M. L. et al. Adolescent Type 1 Diabetes Cardio-Renal Intervention Trial (AdDIT): urinary screening and baseline biochemical and cardiovascular assessments. Diabetes Care 37, 805-13 (2014).

66. Benitez-Aguirre, P. Z. et al. The Adolescent Cardio-Renal Intervention Trial (AdDIT): retinal vascular geometry and renal function in adolescents with type 1 diabetes. Diabetologia 61, 968-976 (2018).

67. Cho, Y. H. et al. Cardiac autonomic dysfunction is associated with high-risk albuminto-creatinine ratio in young adolescents with type 1 diabetes in AdDIT (adolescent type 1 diabetes cardio-renal interventional trial). Diabetes Care 38, 676-81 (2015).

68. Maftei, O. et al. Early atherosclerosis relates to urinary albumin excretion and cardiovascular risk factors in adolescents with type 1 diabetes: Adolescent type 1 Diabetes cardio-renal Intervention Trial (AdDIT). Diabetes Care 37, 3069-75 (2014).

69. Marcovecchio, M. L. et al. Renal and Cardiovascular Risk According to Tertiles of Urinary Albumin-to-Creatinine Ratio: The Adolescent Type 1 Diabetes Cardio-Renal Intervention Trial (AdDIT). Diabetes Care 41, 1963-1969 (2018).

70. ACE Inhibitors in Diabetic Nephropathy Trialist Group. Should all patients with type 1 diabetes mellitus and microalbuminuria receive angiotensin-converting enzyme inhibitors? A meta-analysis of individual patient data. Ann. Intern. Med. 134, 370-9 
(2001).

71. Wiegman, A. et al. Efficacy and safety of statin therapy in children with familial hypercholesterolemia: a randomized controlled trial. JAMA 292, 331-7 (2004).

72. de Boer, I. H. et al. Albuminuria Changes and Cardiovascular and Renal Outcomes in Type 1 Diabetes: The DCCT/EDIC Study. Clin. J. Am. Soc. Nephrol. 11, 1969-1977 (2016).

73. Rapoff, M. Adherence to pediatric medical regimens. (Kluwer Academic/Plenum Publishers, 1999).

74. Benitez-Aguirre P.Z. et al. Top tertile urinary albumin creatinine ratio (ACR)doubles the risk of 3-step progression of diabeticretinopathy: the adolescent type 1 diabetes cardio-renal intervention trial (AdDIT). Pediatr. Diabetes 19, 98 (2018).

75. Vuorio, A. et al. Statins for children with familial hypercholesterolemia. Cochrane Database Syst. Rev. 7, CD006401 (2017).

76. Kuriyama, S. Protection of the kidney with sodium-glucose cotransporter 2 inhibitors: potential mechanisms raised by the large-scaled randomized control trials. Clin. Exp. Nephrol. (2018). doi:10.1007/s10157-018-1673-0 


\section{Box 1. Definitions of microalbuminuria}

Based on albumin-creatine ratio (ACR) in an early morning urine sample:

- Cut-offs used in the ORPS-NFS-AdDIT cohorts:

$3.5-35 \mathrm{mg} / \mathrm{mmol}$ in males

$4.0-47 \mathrm{mg} / \mathrm{mmol}$ in females

in at least two out of three consecutive samples

- Cut-offs used in the international guidelines*:

2.5 to $25 \mathrm{mg} / \mathrm{mmol}$ in males

3.5 to $25 \mathrm{mg} / \mathrm{mmol}$ in females

in at least two out three samples collected over a 3- to 6-month period

Based on albumin excretion rate (timed overnight or 24-hour urine collection)

$20-200 \mu \mathrm{g} / \mathrm{min}$ or $30-300 \mathrm{mg} /$ day

*in the most recent international guidelines the term 'microalbuminuria' has been replaced by 'albuminuria' (ref 21)

ORPS: Oxford Regional Prospective Study; NFS: Nephropathy Family Study; AdDIT: Adolescent Type 1 Diabetes Intervention Trial (refs 31,61, 62) 
Figure 1. Development and Progression of vascular complications of childhood-onset T1DM

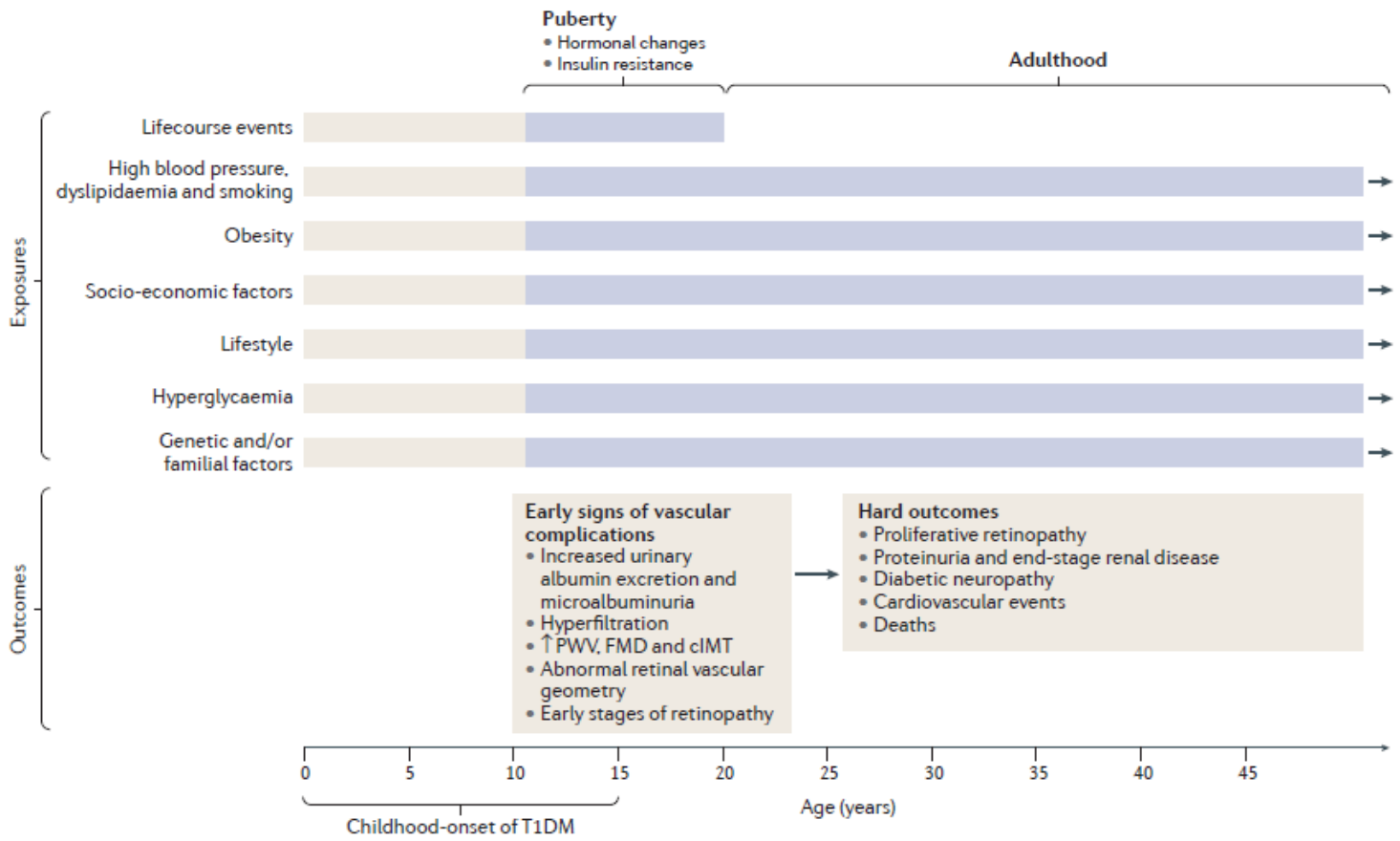

This graph shows the main factors (exposure) contributing to the development of early signs of vascular complications during puberty, and their continuing effect also during adulthood, when overt manifestations (hard outcomes) of vascular complications occur. The graph highlights two unique pubertal contributors, represented by hormonal changes and insulin resistance. PWV: pulse wave velocity, FMD: flow mediated dilation, cIMT: carotid intimamedia thickness 
Figure 2. Urinary albumin excretion changes from the onset of T1DM

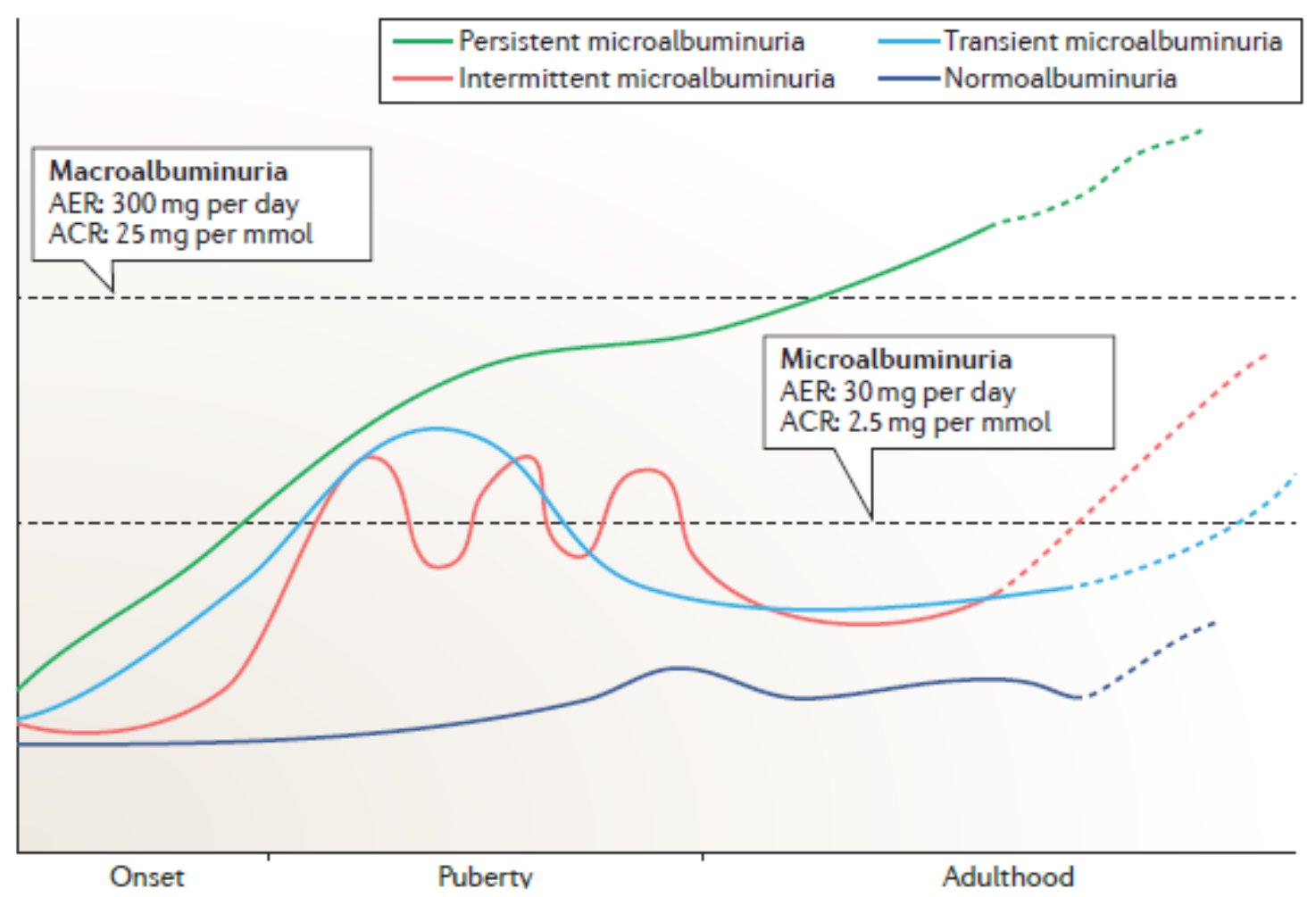

This graph shows four different broad trends for urinary albumin excretion levels identified in children with type 1 diabetes mellitus (T1DM): persistent microalbuminuria, intermittent microalbuminuria, transient microalbuminuria and normoalbuminuria. Continuous lines show trends during childhood. Dashed lines show potential trend during adult life. ACR: albumin-creatinine ratio; AER: albumin excretion rate 
Figure 3. Schematic representation of the design of the AdDIT studies

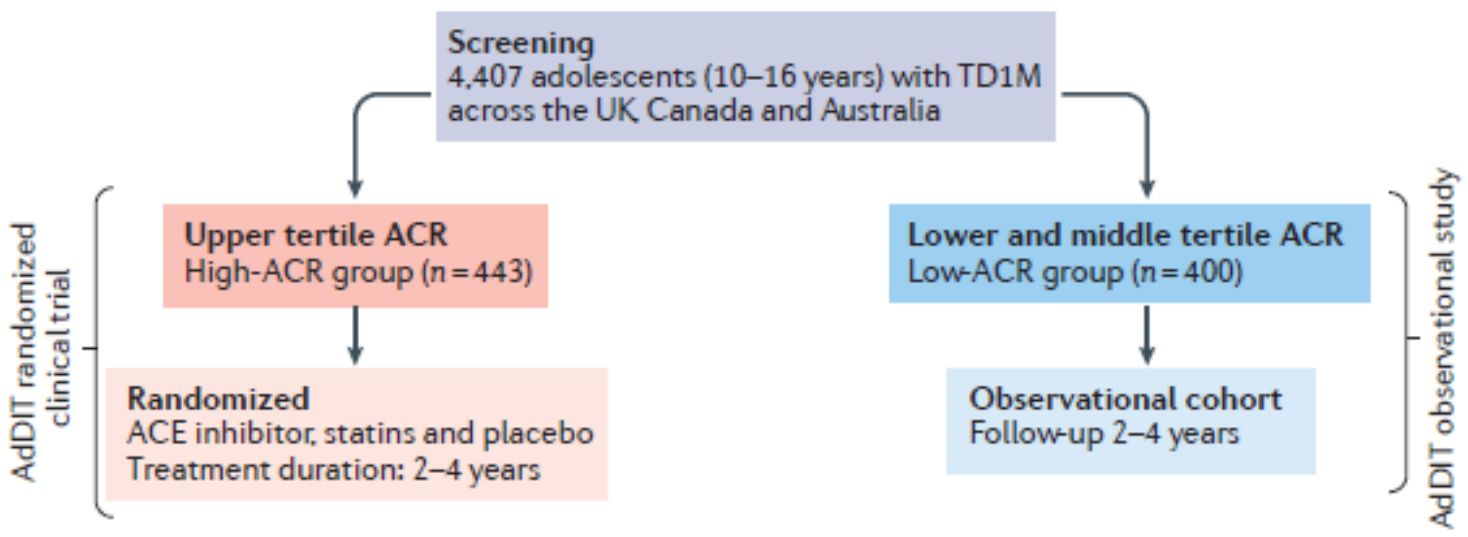

Baseline and follow-up assessments:

1. Clinical data, $\mathrm{HbA}_{1}$

2. Renal markers: urinary ACR cystatin $C$ and creatinine

3. Cardiovascular markers: lipid profile and hsCRP

4. Cardiovascular direct measures: PWV, FMD and cIMT

5. Retinopathy

This graph shows the design of the AdDIT studies, starting from the screening phase, followed by stratification of eligible participants into tertiles of albumin-creatinine ratio (ACR). 443 adolescents with an ACR in the upper tertile (high-ACR group) entered the AdDIT randomized clinical trial and were treated with an ACE-inhibitor+ placebo, a statin+placebo or a combination of both drugs or placebos, whereas 400 adolescents with an ACR either in lower or middle tertile (low-ACR group) were recruited into the AdDIT observational study. Assessment performed at baseline and during the follow up period (duration 2-4years) are reported. PWV: pulse wave velocity, FMD: flow mediated dilation, cIMT: carotid intimamedia thickness 
Figure 4. Cumulative incidence of microalbuminuria by tertiles of $\mathrm{HbA}_{1 \mathrm{c}}$ in the AdDIT cohort

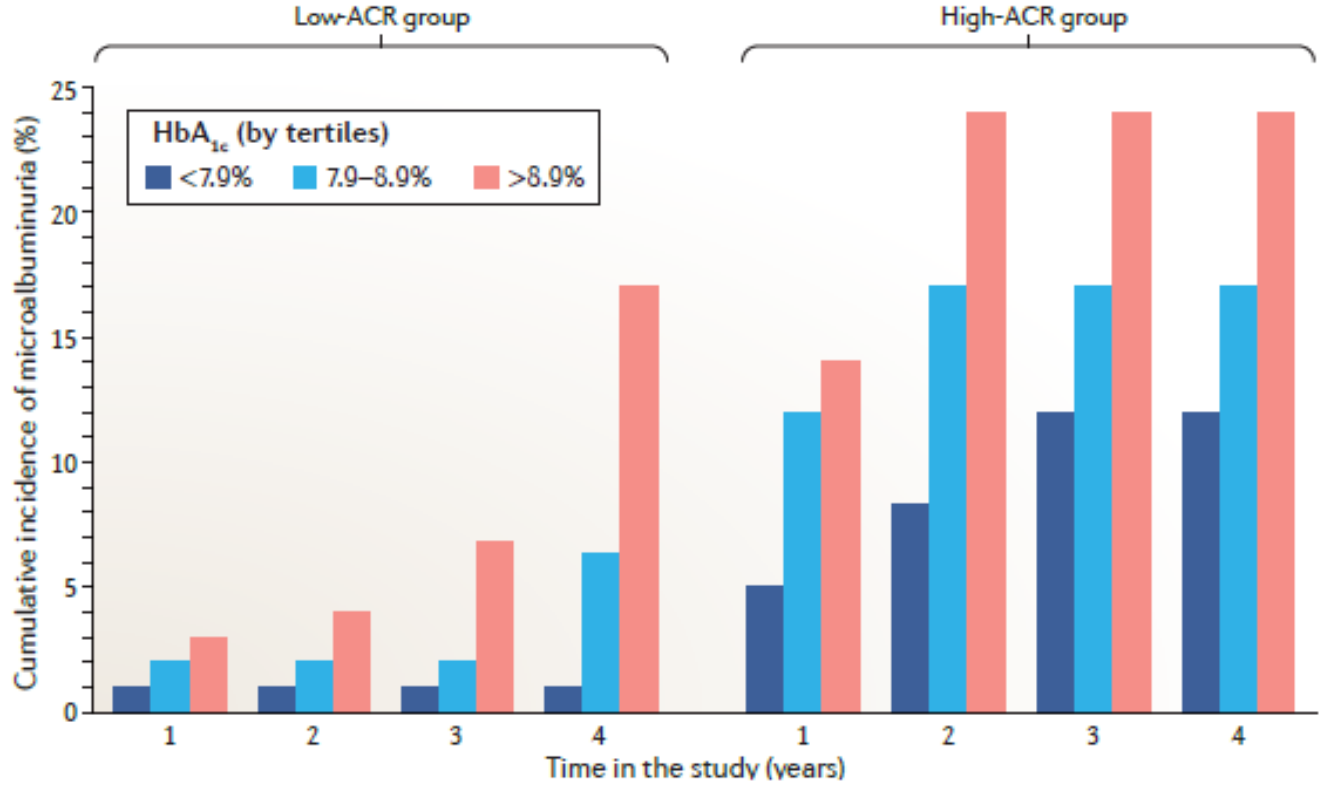

Low ACR (middle and lower tertiles) versus high-ACR (upper tertile). Upper tertile of ACR is defined as an adjusted ACR above a cutoff of log 1.2; middle tertile is between 0.8-1.2 and lower tertile is below 0.8 (ref 65 ). $\mathrm{HbA}_{1 \mathrm{c}}$ levels are expressed as percentages. Adapted with permission from ref 69. 